\title{
THE CONFERENCE ON FIBER BUNDLES AND DIFFERENTIAL GEOMETRY IN ITHACA
}

A conference with the title Fiber bundles and differential geometry was held at Cornell University from May 3 to 7, 1953. It was supported by a grant from the National Science Foundation to Cornell University. The members of the organizational committee were S. S. Chern, University of Chicago, Samuel Eilenberg, Columbia University, N. E. Steenrod (chairman), Princeton University, R. J. Walker, Cornell University, and Hassler Whitney, Institute for Advanced Study.

In addition to the committee and the mathematicians at Cornell, the following twenty-two mathematicians attended the conference on invitation of the committee:

José Adem-Chahin, Warren Ambrose, Armand Borel, Eugenio Calabi, Alex Heller, F. E. P. Hirzebruch, S. T. Hu, Kunihiko Kodaira, E. G. Kundert, S. D. Liao, W. S. Massey, Deane Montgomery, J. C. Moore, G. D. Mostow, Katsumi Nomizu, Everett Pitcher, Hans Samelson, D. C. Spencer, P. A. Smith, R. L. Taylor, H. C. Wang, and G. W. Whitehead.

The program of the conference consisted of eight sessions with talks as follows:

1. Hans Samelson: Survey of spectral sequences and the homology theory of bundles.

W. S. Massey: Exact couples.

A. S. Shapiro: Torsion in fiber bundles.

2. Samuel Eilenberg: Survey of the homology theory of groups and recent results on $A(\pi, n), K(\pi, n)$.

N. E. Steenrod: Reduced powers, and homology theory of the symmetric groups.

José Adem-Chahin: Relations on iterated reduced powers.

3. J. C. Moore: Triad groups and the generalized isomorphism theorem of Hurewicz.

G. W. Whitehead: Survey of the methods of computing homotopy groups of spheres.

Hans Samelson: Pontrjagin products in spaces of loops.

4. Paul Olum: Survey of obstruction theory.

S. D. Liao: The secondary obstruction in sphere bundles.

N. E. Steenrod: The results of Thom and Wu on characteristic classes.

5. Armand Borel: Survey of the cohomology of Lie groups and their coset spaces. 
Alex Heller: Universal bundles in singular homology.

G. D. Mostow: Solvable Lie groups and solvmanifolds.

6. Warren Ambrose: Generalized affine connections.

S. S. Chern: Infinite continuous pseudo-groups.

7. D. C. Spencer: Cohomology and Cousin's problem.

Kunihiko Kodaira: On complete continuous systems of divisors on algebraic varieties.

F. E. P. Hirzebruch: Complex structures on $S^{2} \times S^{2}$.

8. S. S. Chern: The algebraic nature of the Chern classes.

F. E. P. Hirzebruch, Kunihiko Kodaira, and E. G. Kundert: Three talks on "Arithmetic genera."

H. C. Wang: Closed manifolds with homogeneous complex structures.

Some 17 years ago when Whitney isolated the concept of fiber bundle and observed its omnipresence throughout geometry, it became clear that he had opened a path for the large scale application of algebraic topology to the other branches of geometry. The conference program constituted a survey of the very extensive developments of the last six years based on this concept. The first half of the program was devoted to the upheaval within topology itself resulting from the use of fiber space techniques. The second half reviewed the applications to Lie groups, differential geometry, complex analytic manifolds, and algebraic geometry. The most striking feature of the conference was the frequent use of the same apparatus in two or more widely separated disciplines, with strong suggestions of a probable unification of geometry on some higher level. The discussions were marked by the presentations of numerous unsolved problems. These were recorded and a report on them is being prepared for publication.

N. E. Steenrod 